\title{
CONTROLE DA GERMINAÇÃO DE SEMENTES DE SOJA EM TESTES DE SANIDADE PELO USO DA RESTRIÇÃO HÍDRICA ${ }^{1}$
}

\author{
JOSÉ DA CRUZ MACHADO²; JOÃO ALMIR DE OLIVEIRA33; MARIA DAS GRAÇAS G.C. VIEIRA4; \\ MARCELO DE C. ALVES ${ }^{5}$
}

\begin{abstract}
RESUMO - A inibição do processo de germinação de sementes em testes de sanidade é de vital importância na avaliação da ocorrência de fungos, especialmente por melhorar a precisão e a operacionalidade da análise. Como existem inconvenientes nos métodos tradicionais para inibição da germinação nos testes de sanidade, o presente trabalho teve como objetivo investigar a viabilidade da aplicação da restrição hídrica para inibir a germinação de sementes de soja, em substituição ao método tradicional com 2,4,D. Para tanto, foi utilizado como substrato papel de filtro umedecido com soluções de 2,4D (10ppm), e de manitol, $\mathrm{NaCl}$ e $\mathrm{KCl}$ nos potenciais hídricos de 0, -0,4, -06, -08, e -1,0MPa. Pelos resultados, pôde-se verificar que houve inibição crescente da germinação com o aumento da restrição hídrica, não sendo afetadas as incidências dos principais fungos presentes nas amostras analisadas, havendo entretanto melhor controle nas concentrações acima de -0,6 MPa.
\end{abstract}

Termos para indexação: Glycine max, teste de sanidade, restrição hídrica, germinação.

\section{CONTROL OF SEED GERMINATION IN BLOTTER TEST THROUGH THE WATER RESTRICTION TECHNIQUE}

\begin{abstract}
The inhibition of seed germination in health testing is important because germinated seeds may cause contamination and make microscopic assessment difficult. To inhibit seed germination of some dicotyledons species the use of 2,4-D is commonly recommended and for monocotyledons the use of deep freezing is used. In both cases, there are serious inconveniences for several reasons. In the present study the use of the water restriction technique was investigated as mean to inhibit soybean seeds in substitution to 2,4-D in the blotter test. Manitol, $\mathrm{NaCl}$ and $\mathrm{KCl}$ were the osmotic compounds tested. The effects of the water restriction were also evaluated on the occurrence of the fungi associated with seeds. Water restriction in the range of $-0,6$ to $-1,0$ MPa was effective in the inhibition of the seed germination without affecting the occurrence of the fungi in the samples used.
\end{abstract}

Index terms: Glycine max, seed health, water restriction, seed germination.

\section{INTRODUÇÃO}

O teste de sanidade de sementes tem como objetivo determinar a condição sanitária de um lote de sementes, fornecendo informações para programas de certificação, serviços de vigilância vegetal, tratamento de sementes, melhoramento de plantas e outros (Henning, 1994; Machado, 2000).

\footnotetext{
${ }^{1}$ Aceito para publicação em 26/11/2003.

2 Eng $^{\circ}$ Agr $^{\circ}$, Ph.D, Professor do Departamento de Fitopatologia/UFLA; email:machado@ufla.br

${ }^{3}$ Biólogo, Dr., Professor do Departamento de Agricultura/UFLA.

${ }^{4}$ Eng $^{\circ}$ Agr $^{\circ}$, Dr ${ }^{\text {a }}$, Professora do Departamento de Agricultura/UFLA.

${ }^{5}$ Mestrando em agronomia Fitopatologia/UFLA.
}

A germinação rápida das sementes, durante o período de incubação em substrato de papel (blotter test), prejudica a avaliação dos fungos presentes nas sementes e pode comprometer a validade dos resultados devido a possíveis contaminações secundárias entre as sementes e o exterior do recipiente. No intuito de inibir a germinação das sementes, são recomendados, em geral, o uso do herbicida 2,4-D (2,4diclorofenoxiacetato de sódio) e o método do congelamento para sementes de dicotiledôneas e de monocotiledôneas, respectivamente, (Brasil, 1992; Machado, 1988). O 2,4-D apresenta alguns inconvenientes como efeito fungitóxico, caso utilizado em altas concentrações, e por constituir sério risco à saúde dos seus operadores em laboratório. Já, pelo método 
de congelamento, criam-se condições favoráveis, em muitos casos, para o desenvolvimento de bactérias e fungos saprofíticos (Limonard, 1968), além dos custos envolvidos na aquisição de equipamentos de congelamento e a limitação do fluxo de análise de um maior número de amostras que devem ser submetidas a esse processo em um curto período de tempo.

A técnica da restrição hídrica tem sido utilizada, com sucesso, em substituição ao 2,4-D e ao congelamento para sementes de arroz e de feijão (Coutinho, 2000); verificou-se que a restrição hídrica, induzida por $\mathrm{NaCl}, \mathrm{KCl}$ e manitol, em diferentes potenciais osmóticos e em substrato de papel e meio agarizado, não interferiu na detecção dos fungos associados às sementes ou às plântulas. Também foi constatada ausência de efeito no crescimento de alguns dos principais fungos que se associam às sementes de arroz e de feijão quando se modificou o meio BDA, com os mesmos potenciais osmóticos testados para inibir a germinação das sementes.

Desse modo, o presente trabalho teve como objetivo estudar a viabilidade do uso de manitol, $\mathrm{NaCl}$ e $\mathrm{KCl}$ como restritores hídricos para inibir a germinação de sementes de soja, bem como a sua influência sobre a incidência dos fungos durante o teste de sanidade pelo método de incubação em substrato de papel.

\section{MATERIAL E MÉTODOS}

O experimento foi realizado no laboratório de Patologia de Sementes do Departamento de Fitopatologia da Universidade Federal de Lavras-MG.

Foram utilizados dois lotes de sementes de soja, das cultivares, DOKO e IAC-8, produzidas em área experimental da Universidade Federal de Lavras .

Como restritores hídricos foram utilizados os produtos manitol, cloreto de potássio $(\mathrm{KCl})$ e cloreto de sódio $(\mathrm{NaCl})$, nos potenciais hídricos de $0,-0,4,-0,6,-0,8$ e -1,0 MPa e como testemunha referencial solução de 2,4 diclorofenoxiacetato de sódio (2,4-D) a 10 ppm.

As soluções de Manitol, $\mathrm{KCl}$ e $\mathrm{NaCl}$, foram preparadas utilizando-se a fórmula proposta por Van’t Hoff citado por Salisbury e Ross, 1991:

$$
\psi_{\text {os }}=-\mathrm{i} \text { RTC }
$$

sendo: $\psi_{\text {os }}=$ Potencial osmótico $(\mathrm{Mpa})$

$\mathrm{i}^{\mathrm{os}}=$ Coeficiente isotônico,

$\mathrm{R}=$ Constante geral dos gases perfeitos (0,0083 Mpa $\mathrm{x} 1 \mathrm{x} \mathrm{mol}^{-1} \times \mathrm{K}^{-1}$ )

$$
\mathrm{T}=\text { Temperatura absoluta }\left({ }^{\circ} \mathrm{K}\right)
$$$$
\mathrm{C}=\text { Concentração (mol/L) }
$$

As quantidades de cada soluto calculadas foram colocadas em frascos contendo 300mL de água destilada, sendo estes em seguida, esterilizados a $120^{\circ} \mathrm{C}$ por 20 minutos.

Como substrato foram utilizadas três folhas de papel de filtro esterilizadas e umedecidas com cada solução e acondicionadas em placas de Petri de $15 \mathrm{~cm}$ de diâmetro. Para cada soluto em cada potencial, as sementes foram distribuídas, uniformemente, sobre o substrato de papel, em oito repetições representadas por uma placa com 25 sementes, 200 sementes por tratamento.

Após a semeadura as placas foram distribuídas, aleatoriamente, na câmara de incubação com temperatura de $20^{\circ} \mathrm{C} \pm$ $2^{\circ} \mathrm{C}$ e mantidas por 7 dias sob regime alternado de $12 \mathrm{~h}$ de luz e $12 \mathrm{~h}$ de escuro. Após este período, foram feitas as seguintes avaliações:

- Incidência de fungos - a avaliação foi realizada, em cada semente, anotando-se todos os fungos encontrados, sendo a identificação realizada por meio de microscópio estereoscópico com auxílio do microscópio composto.

- Comprimento de radícula das plântulas - após a realização do teste de sanidade foi avaliado, também, o comprimento de radícula das plântulas germinadas; cada plântula foi medida, separadamente, e, em seguida, calculado o comprimento médio das raízes por tratamento.

O delineamento experimental utilizado nos ensaios foi o inteiramente casualisado, utilizando-se análise de regressão para as fontes de variação de $\mathrm{NaCl}, \mathrm{KCl}$ e manitol. O ajuste foi realizado com auxílio do procedimento REG do SAS ${ }^{\circledR}$ (SAS Institute Inc. 1992). Para os tratamentos adicionais, quando se utilizou o 2,4-D ou o método do congelamento, foi determinada a média das repetições destes tratamentos para comparação com os tratamentos utilizados.

\section{RESULTADOS E DISCUSSÃO}

Os resultados referentes ao comprimento de radícula das plântulas oriundas de sementes de soja submetidas à análise sanitária, com restrição hídrica, encontram-se na Figura 1, e os percentuais de fungos detectados nessas sementes são apresentados na Tabela 1.

Conforme a Figura 1, constatou-se, mesmo no menor potencial hídrico (-0,4MPa), independente do soluto utilizado, redução do comprimento de radícula quando comparado às sementes que foram incubadas em substrato umedecido com água pura sem restrição hídrica $(0 \mathrm{MPa})$ e aquelas sub- 
Manitol-Y= 1,463115+(3,72423+1,337494)*X+(4,919062+1,155955)* $X^{\wedge} 2+(2,581061-0,120473) * X^{\wedge} 3$

$\mathrm{KCL}-\mathrm{Y}=1,463115+(3,72423-4,956261) * \mathrm{X}+(4,919062-12,46934) * \mathrm{X}^{\wedge} 2+(2,581061-7,428447) * \mathrm{X}^{\wedge} 3$

$\mathrm{NaCl}-\mathrm{Y}=1,463115+(3,72423 * \mathrm{X})+\left(4,919062 * \mathrm{X}^{\wedge} 2\right)+\left(2,581061 * \mathrm{X}^{\wedge} 3\right)$

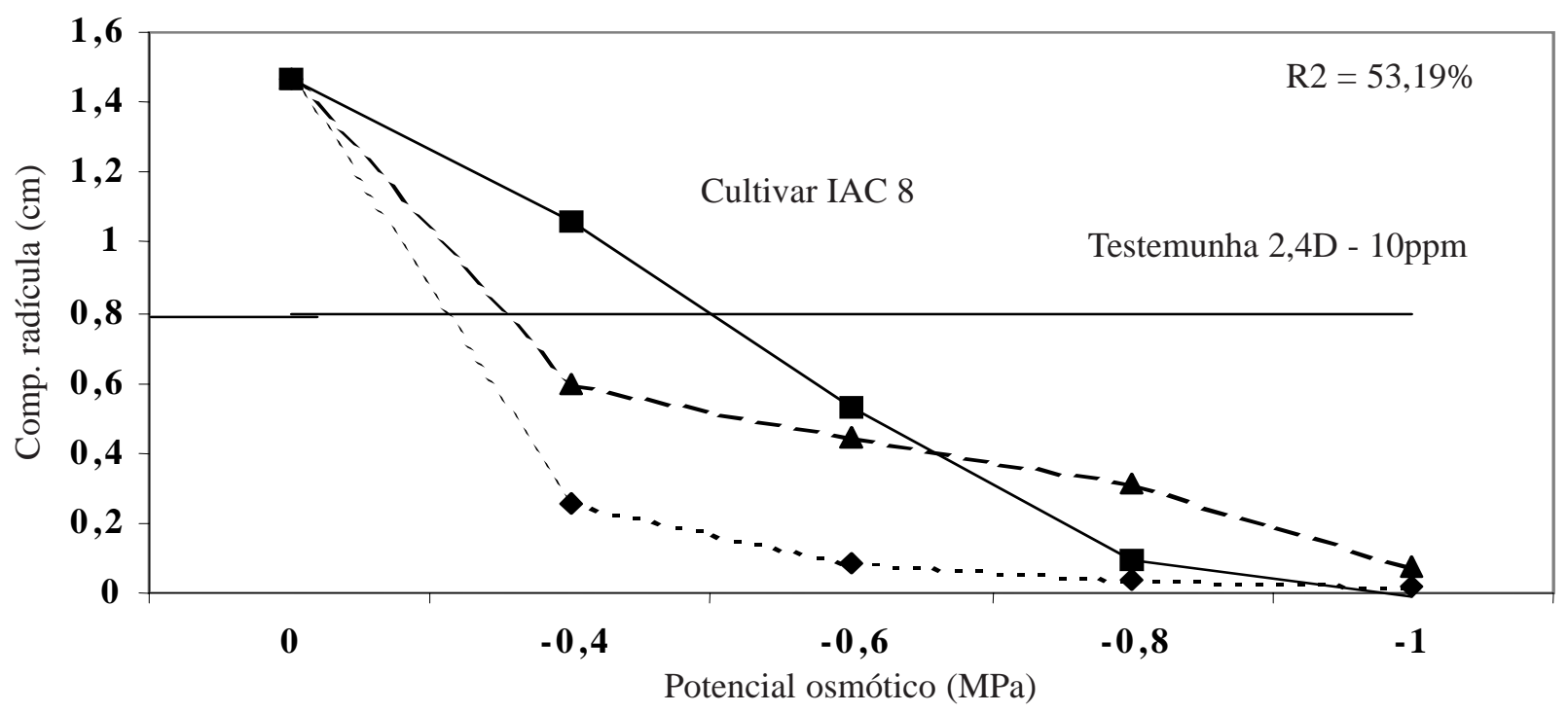

Manitol-Y= 1,629815+(2,88437-1,785172)*X+(0,333873-4,221533)*X^2+(-0,942265-2,467738)* $\mathrm{X}^{\wedge} 3$

$\mathrm{KCL}-\mathrm{Y}=1,629815+(2,88437+1,515615) * \mathrm{X}+(0,333873+3,851046) * \mathrm{X}^{\wedge} 2+(-0,942265+2,367296) * \mathrm{X}^{\wedge} 3$

$\mathrm{NaCl}-\mathrm{Y}=1,629815+(2,88437 * \mathrm{X})+\left(0,333873 * \mathrm{X}^{\wedge} 2\right)+\left(-0,942265 * \mathrm{X}^{\wedge} 3\right)$

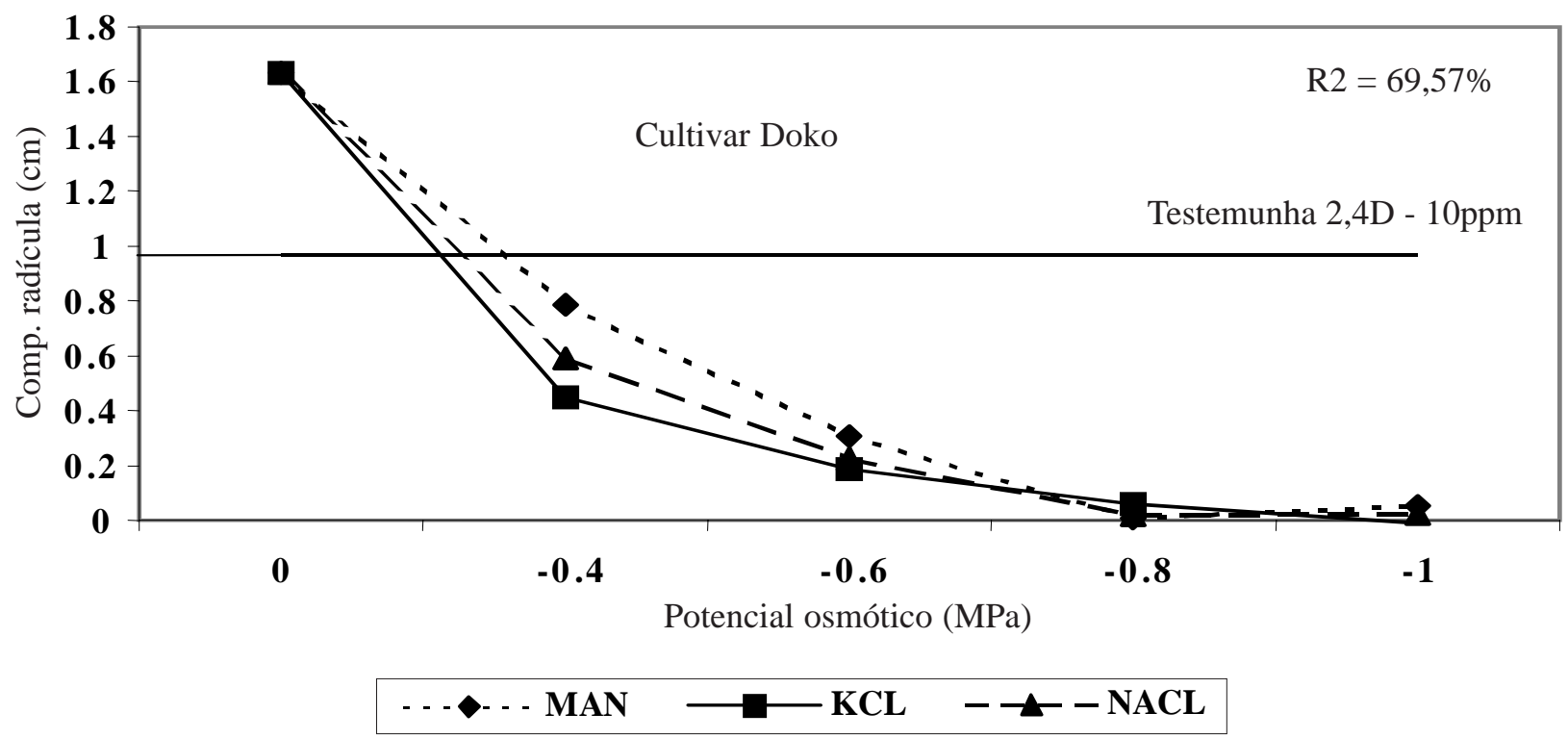

FIG. 1. Comprimento médio de radícula de plântulas oriundas de sementes de soja, cultivares DOKO e IAC-8, submetidas à análise sanitária pelo método de incubação em substrato de papel, com restrição hídrica induzida pela adição de Manitol, NaCl e KCl, em diferentes potenciais e pelo uso de 2,4-D a 10 ppm (testemunha referencial). UFLA, Lavras, MG, 2001. 
TABELA 1. Dados percentuais médios de incidência de fungos em sementes de soja, cultivares IAC-8 e DOKO, submetidas ao teste de sanidade pelo método de incubação em substrato de papel (Blotter Test), sem restrição hídrica (P), com 2,4-D e com restrição hídrica utilizando manitol $\mathrm{KCl}$ e $\mathrm{NaCl}$ em diferentes concentrações. UFLA-Lavras (MG), 2001.

\begin{tabular}{|c|c|c|c|c|c|c|c|c|c|c|c|c|c|c|c|}
\hline \multirow{3}{*}{ Cultivar } & \multirow{3}{*}{ Fungos } & \multirow{3}{*}{$2,4 \mathrm{D}$} & \multirow{3}{*}{$\mathbf{P}$} & \multicolumn{12}{|c|}{ Potencial (MPa) } \\
\hline & & & & \multicolumn{4}{|c|}{ Manitol } & \multicolumn{4}{|c|}{$\mathrm{KCl}$} & \multicolumn{4}{|c|}{$\mathrm{NaCl}$} \\
\hline & & & & $-0,4$ & $-0,6$ & $-0,8$ & -1 & $-0,4$ & $-0,6$ & $-0,8$ & -1 & $-0,4$ & $-0,6$ & $-0,8$ & -1 \\
\hline \multirow[t]{7}{*}{ IAC-8 } & Asp. spp. & 0,5 & 0,5 & 2 & 0,5 & 1 & 1 & 0 & 0 & 0 & 1 & 0,5 & 0 & 0 & 0,5 \\
\hline & Cercospora kikuchii & 12,5 & 17,5 & 16 & 12 & 10,5 & 8,5 & 10,5 & 15,5 & 10 & 9 & 11 & 14 & 6,5 & 13 \\
\hline & Cladosporium cladosporioides & 66,5 & 56 & 60,5 & 59 & 55,5 & 59 & 58 & 63 & 50,5 & 53 & 58 & 50,5 & 50 & 63 \\
\hline & Colletotrichum truncatum & 0 & 0,5 & 0 & 0 & 0 & 0 & 0 & 0,5 & 0,5 & 0 & 0 & 0 & 0 & 0 \\
\hline & Fusarium & 7,5 & 8,5 & 2,5 & 0,5 & 4 & 4 & 4 & 2 & 4 & 8 & 7,5 & 6 & 8,5 & 11,5 \\
\hline & Phomopsis sojae & 17 & 25,5 & 24 & 16,5 & 20 & 17 & 16,5 & 18 & 11,5 & 14 & 20 & 8,5 & 5,5 & 10,5 \\
\hline & Penicillium & 10 & 13,5 & 13 & 9,5 & 12 & 9,5 & 11 & 16,5 & 9 & 10 & 11 & 15,5 & 14 & 14 \\
\hline \multirow[t]{7}{*}{ DOKO } & Asp. spp. & 0 & 3 & 1,5 & 2 & 1 & 0 & 1 & 0,5 & 1 & 1,5 & 0,5 & 2,5 & 0,5 & 1 \\
\hline & Cercospora kikuchii & 25 & 19,5 & 14 & 20 & 11,5 & 25 & 25 & 23,5 & 23 & 22 & 27,5 & 23,5 & 27 & 20,5 \\
\hline & Cladosporium cladosporioides & 75 & 59 & 76 & 69 & 67,5 & 79,5 & 68 & 67,5 & 76,5 & 76,5 & 68 & 69 & 72 & 76 \\
\hline & Colletotrichum .truncatum & 0 & 0 & 0 & 0 & 0 & 0 & 0,5 & 0 & 0 & 0,5 & 0 & 0 & 0 & 0 \\
\hline & Fusarium & 8 & 16,5 & 18,5 & 17 & 8 & 16 & 8,5 & 6,5 & 6 & 11,5 & 15 & 16 & 11,5 & 10 \\
\hline & Phomopsis sojae & 4,5 & 8 & 3,5 & 5,5 & 3,5 & 6,5 & 5,5 & 4 & 2 & 3,5 & 6 & 2 & 4,5 & 2,5 \\
\hline & Penicillium & 1 & 3 & 3,5 & 5,5 & 4,5 & 5 & 3,5 & 2 & 4,5 & 1 & 4,5 & 3 & 3,5 & 5 \\
\hline
\end{tabular}

metidas ao tratamento com o herbicida (2,4-D). Já nos potenciais de -0,8 e -1,0 MPa o efeito foi maior do que os demais tratamentos, chegando inclusive à inibição quase completa da emissão da rádicula. Em trabalho de Braccini et al. (1996), reduções da germinação e do vigor de sementes de soja foram constatados em potenciais mais negativos que -0,3MPa induzidos por soluções de $\mathrm{NaCl}$, manitol e PEG 6000. A solução de $\mathrm{NaCl}$ na concentração de -0,9 $\mathrm{MPa}$, afetou severamente a emissão da radícula. Também Coutinho (2000), em trabalho com sementes de arroz e de feijão, utilizando como restritores hídricos manitol, $\mathrm{NaCl}$ e $\mathrm{KCl}$, verificou efeito inibidor crescente na emissão da radícula com a elevação da restrição hídrica do substrato.

Em estudos sobre inoculação de Colletotrichum lindemuthianum em sementes de feijão, Carvalho (1999) verificou ausência de protrusão radicular nas sementes mantidas por 120 horas em substrato umedecido com manitol na concentração de -1,0MPa.

Os resultados constantes da tabela 1 , referentes ao teste de sanidade de sementes de soja, revelaram, de modo geral, ausência de diferenças marcantes entre os diferentes tipos de restritores hídricos e potenciais utilizados, quando comparados aos do método de 2,4-D e com a testemunha, sem inibição da germinação. As pequenas diferenças podem ser atribuídas à amostragem, sendo comumente observadas mesmo entre repetições de um mesmo teste; verificou-se, também, que essas variações ocorreram mesmo dentro de cada soluto utilizado. Estes resultados são constantes àqueles obtidos por Coutinho (2000) que, trabalhando com os mesmos restritores hídricos no teste de sanidade de sementes de arroz e de feijão, não contatou diferenças significativas entre os resultados quando comparados com a metodologia tradicional (congelamento ou 2,4-D).

\section{CONCLUSÕES}

A utilização da restrição hídrica, por meio dos solutos: Manitol, $\mathrm{NaCl}$ e $\mathrm{KCl}$ em potenciais de -0,6 a -1,0 MPa, revela se como um procedimento promissor para impedir ou reduzir a germinação de sementes de soja por ocasião do teste de incubação em substrato de papel visando à detecção dos principais fungos associados às sementes desta espécie. 


\section{REFERÊNCIAS}

BRASIL. Ministério da Agricultura e Reforma Agrária. Regras para análise de sementes. Brasília, 1992. 365p.

BRACCINI, A. de L.; RUIZ, H.A; BRACCINI, M.C.L.; REIS, M.S. Germinação e vigor de sementes de soja sob estresse hídrico induzido por soluções de cloreto de sódio, manitol e polietileno glicol. Revista Brasileira de Sementes, Brasília, v.18, n.1, p.10-16, 1996.

CARVALHO, J.C.B. Uso da restrição hídrica na inoculação de Colletotrichum lindemuthianum em sementes de feijoeiro (Phaseolus vulgaris L. ). Lavras: UFLA, 1999. 98p. (Dissertação de mestrado).

COUTINHO, W.C. Uso da restrição hídrica no controle da germinação de sementes de arroz (Oryza sativa $\mathrm{L}$.) e feijoeiro (Phaseolus vulgaris L.) em testes de sanidade. Lavras: UFLA, 2000. 78p. (Dissertação de mestrado).
HENNING, A.A. Patologia de Sementes. Londrina: EMBRAPA CNPSo, 1994. 43p. (EMBRAPA - CNPSo / Documento 90).

LIMONARD, T. Ecological aspects of seed health testing. Proceedings of International Seed Testing Association, Wageningen, v.33, n.3, p.343-513, 1968.

MACHADO, J. da C. Patologia de sementes: fundamentos e aplicações. Brasília: Ministério da Educação, ESAL: FAEPE, 1988. 107p.

MACHADO, J. da C. Tratamento de sementes no controle de doenças. Lavras: LAPS/UFLA/FAEPE, 2000. 138p.

SALISBURY, F.B.; ROSS, C.W. Plant Physiology. 4 ed., Belmont. Wadsworth, 1991. 682p.

SAS Institute Inc. SAS technical report SAS / STAT software: changes and enhancement relase 607, Cary Nc: SAS Institute Inc., 1992. 